\title{
Impact of anisotropy on the noncritical squeezing properties of two-transverse-mode optical parametric oscillators
}

\author{
Carlos Navarrete-Benlloch ${ }^{1, *}$ and Germán J. de Valcárcel ${ }^{2}$ \\ ${ }^{1}$ Max-Planck-Institut für Quantenoptik, Hans-Kopfermann-strasse 1, D-85748 Garching, Germany \\ ${ }^{2}$ Departament d'Òptica, Universitat de València, Dr. Moliner 50, E-46100 Burjassot, Spain
}

(Received 13 January 2013; published 19 June 2013)

\begin{abstract}
In a series of articles we studied the quantum properties of a degenerate optical parametric oscillator tuned to the first family of transverse modes at the subharmonic. We found that, for a cavity having rotational symmetry with respect to the optical axis, a TEM 10 mode with an arbitrary orientation in the transverse plane is emitted above threshold. We proved then that quantum noise induces a random rotation of this bright $\mathrm{TEM}_{10}$ mode in the transverse plane, while the orthogonal mode, the so-called dark mode, has perfect quadrature squeezing irrespective of the distance to threshold (noncritical squeezing). This result was linked to the spontaneous rotational symmetry breaking which occurs when the bright mode is generated, and here we analyze how the squeezing of the dark mode is degraded when the cavity is not perfectly symmetric. We will show that large levels of squeezing are still attainable for reasonable values of this anisotropy, with the advantage that the orientation of the bright and dark modes is basically fixed, a very attractive situation from the experimental point of view.
\end{abstract}

DOI: $10.1103 /$ PhysRevA.87.065802

PACS number(s): 42.50.Lc, 42.65.Yj, 42.50.Dv

Introduction and previous results. Squeezed states are a fundamental resource both for high-precision measurements [1-4] and quantum information protocols based on continuous variables $[5,6]$. Nowadays, the highest quality squeezed states are generated by using optical parametric oscillators (OPOs) [7-11], which in essence consist in a second-order nonlinear crystal embedded in an optical cavity. When pumped with a field of frequency $2 \omega_{0}$, photons of frequency $\omega_{0}$ are generated in the crystal via the process of parametric down-conversion, and large levels of squeezing are found in this down-converted field only when the OPO is operated close to threshold [12].

In recent works we have analyzed the properties of such devices when several down-conversion channels corresponding to different temporal [13-15], spatial [14,16-21], or polarization [22] modes, are available for a given pumping scheme, obtaining a so-called multimode OPO. We have shown that the properties of these devices can be understood in terms of two fundamental phenomena [23]: pump clamping [19] and spontaneous symmetry breaking [16-18,20,22].

In a series of papers $[18,20,21]$, we have studied in detail the quantum properties derived from the spontaneous symmetry breaking process by means of a particular example: the two-transverse-mode degenerate OPO. The particularity of this OPO is that, while a $\mathrm{TEM}_{00}$ mode is resonant at frequency $2 \omega_{0}$ as usual, its cavity is tuned to the first family of transverse modes at the subharmonic frequency $\omega_{0}$, so that pump photons can be down-converted into pairs of either $\mathrm{TEM}_{10}$ or $\mathrm{TEM}_{01}$ photons. This configuration has been already tested in several experiments [24-26], although none of them have entered the regime in which the first transverse mode family oscillates above threshold, which could be accomplished via the multiGaussian pump technique introduced in [15].

Classically, when this OPO is pumped above some threshold, we proved that emission takes place in a $\mathrm{TEM}_{10}$ mode with an arbitrary orientation in the transverse plane, this

\footnotetext{
*carlos.navarrete@mpq.mpg.de
}

indeterminacy coming from the invariance of the system under rotations around the optical axis [18,20]. We distinguish then between a bright and a dark mode, which are, respectively, the TEM $_{10}$ mode in which mean-field emission occurs and the orthogonal mode.

As for the quantum phenomena, we showed two fundamental properties [18,20]. First, as any orientation of the bright mode is allowed, quantum noise is able to act freely on this variable; we found that the variance of this orientation increases linearly with time, so that, eventually, the orientation of the mode becomes completely uncertain. On the other hand, while the properties of the bright mode are the same as those of the single-mode DOPO (large squeezing levels working close to threshold), the dark mode was shown to have perfect quadrature squeezing at any pumping level above threshold, a result which we interpreted in terms of an angle-angular momentum uncertainty relation [18,20]. This noncritical squeezing is reminiscent of other phenomena present in OPOs above threshold such as intensity correlations [27,28] or bi- and tri-partite entanglement [29-33].

However, since the orientation of the dark mode is uncertain (in the quantum mechanical sense), it seems highly unlikely to match the local oscillator of a homodyne detection to it [20], and we studied in [21] the possibility of locking its orientation by seeding the cavity with a $\mathrm{TEM}_{10}$ coherent beam at the subharmonic, what constitutes an explicit breaking of the system's symmetry, and hence degrades the level of squeezing found in the dark mode (which corresponds to the $\mathrm{TEM}_{01}$ mode when locking is accomplished). We showed that large levels of squeezing can still be obtained for reasonable values of the injection's power.

In this brief report we study how the quantum properties of this system are affected by two sources of anisotropy: a tilt of the nonlinear crystal (or, equivalently, the introduction of a tilted dielectric slab in the cavity), and a possible astigmatism of the mirrors. The first one is particularly interesting, since it allows the locking of the bright mode's orientation in a noninvasive and controllable way. In addition, a tilting of the 
nonlinear crystal is sometimes desirable for phase-matching purposes [34], and our study will reveal the limits that the degradation of squeezing imposes on this technique. Let us remark that although the crystal birefringence is another common source of anisotropy [35], it plays no role in our case, since the down-converted photons have the same polarization (type I OPO); this interplay between birefringence and anisotropy has been studied in other contexts though, for example, regarding the transfer of orbital angular momentum from the pump to the down-converted field of a type II OPO [36,37], or the hyperentanglement achievable on this system [38].

Describing transverse anisotropy. The fact that both the $\mathrm{TEM}_{10}$ and $\mathrm{TEM}_{01}$ modes (or any $\mathrm{TEM}_{10}$ mode with an arbitrary orientation in the transverse plane) have the same resonant frequency comes from the system's invariance with respect to rotations around the optical axis (taken as the $z$ axis). As we show below, the main effect of any source of transverse anisotropy is the introduction of a detuning between the $\mathrm{TEM}_{10}$ and $\mathrm{TEM}_{01}$ modes. In what follows, we will take $\omega_{0}$ to be the frequency of the $\mathrm{TEM}_{10}$ mode, while the TEM $\mathrm{T}_{01}$ mode will resonate at frequency $\omega_{0}+\Delta$, being $\Delta$ the detuning introduced by the anisotropy. As long as $\Delta / \omega_{0}$ remains small, any other effect such as the change in the spatial form of the modes turns out to be irrelevant for our purposes.

We will consider two different sources of anisotropy. The first one consists in allowing one of the mirrors to have some astigmatism; we model this by introducing different curvature radii $R_{x}$ and $R_{y}$ in the $x$ and $y$ directions, respectively, so that the mirror becomes ellipsoidal. The second one consists in allowing the $\chi^{(2)}$ crystal (of length $l_{\mathrm{c}}$ and refractive index $n_{\mathrm{c}}$ ) to be slightly tilted with respect to the cavity axis; we will assume that tilt occurs in the $z x$ plane with angle $\beta$ with respect to the optical axis, what introduces different effective lengths along the $x$ and $y$ directions given by [39]

$$
\begin{aligned}
& L_{\mathrm{eff}, y}=L-l_{\mathrm{c}}\left[|\cos \beta|-\frac{\cos ^{2} \beta}{\left(n_{\mathrm{c}}^{2}-\sin ^{2} \beta\right)^{1 / 2}}\right], \\
& L_{\mathrm{eff}, x}=L_{\mathrm{eff}, y}-l_{\mathrm{c}} \frac{\left(n_{\mathrm{c}}^{2}-1\right) \sin ^{2} \beta}{\left(n_{\mathrm{c}}^{2}-\sin ^{2} \beta\right)^{3 / 2}},
\end{aligned}
$$

$L$ being the cavity length. Note that instead of tilting the nonlinear crystal, one can obtain the same effect by introducing a tilted dielectric slab with antireflecting coating.

Both types of anisotropies have the effect of changing the $g$ parameters [40] of the cavity in a different way for the $x$ and $y$ transverse directions, hence turning the cavity into a composition of two orthogonal one-dimensional (1D) cavities with $g$ parameters $g_{1 x}=1-L_{\text {eff }, x} / R\left(g_{2 x}=1-L_{\text {eff }, x} / R_{x}\right)$ and $g_{1 y}=1-L_{\text {eff, } y} / R\left(g_{2 y}=1-L_{\text {eff, } y} / R_{y}\right)$ for mirror 1 (2). Note that we have taken mirror 2 as the astigmatic one for definiteness. The resonance frequency of a $\mathrm{TEM}_{m n}$ mode with longitudinal index $q$ is then given by

$$
\begin{aligned}
\omega_{q m n}= & \frac{\pi c}{L_{\mathrm{opt}}}\left(q+\frac{m+1 / 2}{\pi} \arccos \sqrt{g_{1 x} g_{2 x}}\right. \\
& \left.+\frac{n+1 / 2}{\pi} \arccos \sqrt{g_{1 y} g_{2 y}}\right),
\end{aligned}
$$

where $c$ is the speed of light, $L_{\mathrm{opt}}=L+\left[\left(n_{\mathrm{c}}^{2}-\sin ^{2} \beta\right)^{1 / 2}-\right.$ $|\cos \beta|] l_{\mathrm{c}}$, and where we have assumed that the curvature radii of the mirrors are larger than the corresponding effective lengths as usually happens in OPO experiments, so that all the $g$ parameters are larger than one. Hence the detuning between the $\mathrm{TEM}_{10}$ and $\mathrm{TEM}_{01}$ modes is given by

$$
\Delta=c\left(\arccos \sqrt{g_{1 y} g_{2 y}}-\arccos \sqrt{g_{1 x} g_{2 x}}\right) / L_{\mathrm{opt}} .
$$

The model equations. As usual, the cavity is pumped by an external laser resonant at frequency $2 \omega_{0}$ with a TEM $_{00}$ mode of the cavity; pump photons are down-converted in the $\chi^{(2)}$ crystal into pairs of photons laying either in the $\mathrm{TEM}_{10}$ or $\mathrm{TEM}_{01}$ mode. The (interaction picture) Hamiltonian is written as

$$
\hat{H}=\hbar \Delta \hat{a}_{y}^{\dagger} \hat{a}_{y}+i \hbar \mathcal{E}_{\mathrm{p}} \hat{a}_{0}^{\dagger}+i \hbar \chi \hat{a}_{0}\left(\hat{a}_{x}^{\dagger 2}+\hat{a}_{y}^{\dagger 2}\right) / 2+\text { H.c. },
$$

being $\hat{a}_{0}, \hat{a}_{x}$, and $\hat{a}_{y}$ the annihilation operators for $\mathrm{TEM}_{00}$, $\mathrm{TEM}_{10}$, and $\mathrm{TEM}_{01}$ photons, respectively. $\mathcal{E}_{\mathrm{p}}$ is proportional to the amplitude of the pumping laser, and we take it as real without loss of generality. The nonlinear coupling $\chi$ is proportional to the product of the crystal's nonlinear susceptibility and the overlapping transverse integral between the three modes involved in the particular down-conversion process, and is the same for the $\mathrm{TEM}_{10}$ and $\mathrm{TEM}_{01}$ modes except for corrections of order $\Delta / \omega_{0}$, which can be neglected for optical frequencies and reasonable anisotropies.

As usual, we incorporate losses through the partially transmitting mirror by introducing the decay rates $\gamma_{\mathrm{p}}$ and $\gamma_{\mathrm{s}}$ in the master equation for the pump and down-converted modes, respectively. Then we map the master equation into a set of stochastic Langevin equations within the positive $P$ representation; given the resemblance of our Hamiltonian to that of [21], we can just take the Langevin equations of that reference but including a detuning $\Delta$ for the $\mathrm{TEM}_{01}$ mode and no subharmonic injection,

$$
\begin{aligned}
& \dot{\alpha}_{0}=\mathcal{E}_{\mathrm{p}}-\gamma_{\mathrm{p}} \alpha_{0}-\chi\left(\alpha_{x}^{2}+\alpha_{y}^{2}\right) / 2, \\
& \dot{\alpha}_{x}=-\gamma_{\mathrm{s}} \alpha_{x}+\chi \alpha_{0} \alpha_{x}^{+}+\sqrt{\chi \alpha_{0}} \eta_{x}(t), \\
& \dot{\alpha}_{y}=-\left(\gamma_{\mathrm{s}}+i \Delta\right) \alpha_{y}+\chi \alpha_{0} \alpha_{y}^{+}+\sqrt{\chi \alpha_{0}} \eta_{y}(t),
\end{aligned}
$$

plus the equations for $\alpha_{m}^{+}$( $\left.m=0, x, y\right)$, which are like those for $\alpha_{m}$ with the changes $\alpha_{m} \longleftrightarrow \alpha_{m}^{+}, \eta_{m}(t) \rightarrow \eta_{m}^{+}(t)$, and $i \rightarrow$ $-i . \eta_{m}(t)$ and $\eta_{m}^{+}(t)$ are real, Gaussian noises with zero mean and whose only nonzero correlations are

$$
\left\langle\eta_{m}(t) \eta_{m^{\prime}}\left(t^{\prime}\right)\right\rangle=\left\langle\eta_{m}^{+}(t) \eta_{m^{\prime}}^{+}\left(t^{\prime}\right)\right\rangle=\delta_{m, m^{\prime}} \delta\left(t-t^{\prime}\right) .
$$

These equations allow us to obtain the noise spectrum of any quadrature $\hat{X}_{m}^{\varphi}=\exp (-i \varphi) \hat{a}_{m}+\exp (i \varphi) \hat{a}_{m}^{\dagger}$ as

$$
V^{\text {out }}\left(\omega ; \hat{X}_{m}^{\varphi}\right)=1+S_{m}^{\varphi}(\omega)
$$

with the squeezing spectrum given by

$$
S_{m}^{\varphi}(\omega)=2 \gamma_{m} \int_{-\infty}^{+\infty} d \tau e^{-i \omega \tau}\left\langle\delta X_{m}^{\varphi}(0) \delta X_{m}^{\varphi}(\tau)\right\rangle .
$$

In this expression $X_{m}^{\varphi}=\exp (-i \varphi) \alpha_{m}+\exp (i \varphi) \alpha_{m}^{+}$is the stochastic counterpart of the corresponding quadrature operator and $\delta X=X-\langle X\rangle$. For vacuum $S_{m}^{\varphi}(\omega)=0$, and hence $V^{\text {out }}\left(\bar{\omega} ; X_{m}^{\varphi}\right)<1$ signals squeezing in the corresponding quadrature at noise frequency $\bar{\omega}$. 


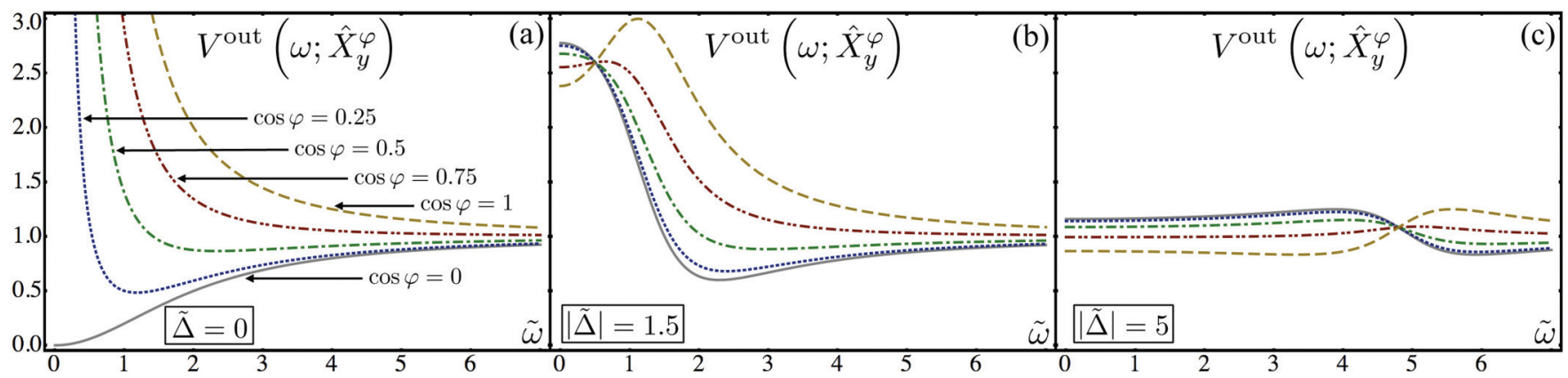

FIG. 1. (Color online) Noise spectrum of the $\mathrm{TEM}_{01}$ quadratures indicated in the plot as a function of the noise frequency. Note that the minimum noise is always achieved in the $\varphi=\pi / 2$ quadrature; interestingly, it is easy to prove from (13) that for $\tilde{\Delta}^{2}>2$ this quadrature has more noise than the $\varphi=0$ quadrature at $\omega=0$, as can be appreciated in (b) and (c). Indeed, the latter can even show squeezing for $\tilde{\Delta}^{2}>4$; in particular, for $4<\tilde{\Delta}^{2}<2(3+\sqrt{5})$ its optimum squeezing is found at $\omega=0$, while for $\tilde{\Delta}^{2}>2(3+\sqrt{5})$ it is found at $\omega^{2}=\tilde{\Delta}^{2}-2|\tilde{\Delta}|-4$, and has the same value as the optimum squeezing in the $\varphi=\pi / 2$ quadrature (c).

The classical picture. The classical dynamic equations of the system are recovered from (5) by removing the noise terms and replacing $\alpha_{m}^{+}$by $\alpha_{m}^{*}$. The resulting equations have two types of stationary solutions as is well known in OPOs: For $\mathcal{E}_{\mathrm{p}}$ below some threshold value the down-converted modes are switched off, while above that threshold one of them is switched on. Which mode wins the nonlinear competition is dictated solely by which one has the lowest threshold [19]. In the current case the $\mathrm{TEM}_{10}$ mode is on resonance and its threshold corresponds to that of the single-mode DOPO, that is, $\mathcal{E}_{\mathrm{th}, x}=\gamma_{\mathrm{p}} \gamma_{\mathrm{s}} / \chi$; on the other hand, the TEM 01 mode is detuned and hence its threshold is given by $\mathcal{E}_{\mathrm{th}, y}=\sqrt{1+\Delta^{2} / \gamma_{\mathrm{s}}^{2}} \mathcal{E}_{\mathrm{th}, x}$ $[41,42]$. Hence, the TEM 10 mode has the lowest threshold, and thus above threshold the only stable, stationary solution is given by

$$
\begin{aligned}
& \bar{\alpha}_{0}=\gamma_{\mathrm{s}} / \chi, \quad \bar{\alpha}_{y}=0, \\
& \bar{\alpha}_{x}= \pm \rho \quad \text { with } \quad \rho=\sqrt{2\left(\mathcal{E}_{\mathrm{p}}-\mathcal{E}_{\text {th }}\right) / \chi},
\end{aligned}
$$

showing that the anisotropy fixes the bright and dark TEM modes to the $x$ and $y$ axis, respectively, what is very convenient from the detection point of view.

Squeezing properties of the dark mode. One of the goals of this work is to show that the quantum properties of the dark mode, which has perfect squeezing irrespective of the distance to threshold in an isotropic cavity, remain useful for reasonable values of the anisotropy. Hence, we study now the squeezing properties of the $\mathrm{TEM}_{01}$ mode, which is the dark mode in the current setup.

As usual, we linearize the Langevin equations (1a) around the classical steady-state solution (1a); in the case of the $\mathrm{TEM}_{01}$ mode this leads to

$$
\dot{\boldsymbol{\alpha}}_{y}=\mathcal{L} \boldsymbol{\alpha}_{y}+\sqrt{\gamma_{\mathrm{s}}} \boldsymbol{\eta}_{y}(t)
$$

with $\boldsymbol{\alpha}_{y}=\operatorname{col}\left(\alpha_{y}, \alpha_{y}^{+}\right), \boldsymbol{\eta}_{y}=\operatorname{col}\left(\eta_{y}, \eta_{y}^{+}\right)$, and

$$
\mathcal{L}=\left(\begin{array}{cc}
-\gamma_{\mathrm{s}}-i \Delta & \gamma_{\mathrm{s}} \\
\gamma_{\mathrm{s}} & -\gamma_{\mathrm{s}}+i \Delta
\end{array}\right)
$$

Following Collett and Walls [43], the squeezing spectrum of any TEM ${ }_{01}$ quadrature can then be obtained as

$$
S_{y}^{\varphi}(\omega)=2 \gamma_{\mathrm{s}} \operatorname{Re}\left\{e^{-2 i \varphi} \mathcal{S}_{11}(\omega)+\mathcal{S}_{12}(\omega)\right\},
$$

where the spectral covariance matrix is given in our case by $\mathcal{S}(\omega)=\gamma_{\mathrm{s}}(\mathcal{L}+i \omega \mathcal{I})^{-1}\left(\mathcal{L}^{T}-i \omega \mathcal{I}\right)^{-1}$, being $\mathcal{I}$ the $2 \times 2$ identity matrix. A straightforward algebraic manipulation allows us to write

$$
S_{y}^{\varphi}(\omega)=\frac{4\left(\tilde{\Delta}^{2}-\tilde{\omega}^{2}\right)+8\left(2-\tilde{\Delta}^{2}+\tilde{\omega}^{2}\right) \cos ^{2} \varphi}{4 \tilde{\omega}^{2}+\left(\tilde{\Delta}^{2}-\tilde{\omega}^{2}\right)^{2}},
$$

where $\tilde{\Delta}=\Delta / \gamma_{\mathrm{s}}$ and $\tilde{\omega}=\omega / \gamma_{\mathrm{s}}$. The corresponding noise spectrum (7) is shown in Fig. 1 for different values of the parameters. A simple inspection of this expression shows that $\varphi=\pi / 2$ is the maximally squeezed quadrature for any combination of the parameters; also, it is straightforward to show that for a given value of the detuning, there exists an optimum detection frequency $\tilde{\omega}_{\text {opt }}^{2}=\tilde{\Delta}^{2}+2|\tilde{\Delta}|$ for which squeezing is maximum, $V_{\mathrm{opt}}^{\text {out }}=|\tilde{\Delta}| /(1+|\tilde{\Delta}|)$ in particular. This expression shows that more than $90 \%$ of squeezing ( $V_{\mathrm{opt}}^{\text {out }}<0.1$ ) can be obtained as long as $|\tilde{\Delta}|<0.1$.

In order to get an idea of the detuning induced by the different sources of anisotropy considered in this brief report, in Fig. 2 we show the dependence of $|\tilde{\Delta}|$ with the tilting angle $\beta$ of the crystal and the ellipticity parameter $\varepsilon=1-$ $R_{y} / R_{x}$ of the astigmatic mirror, for typical OPO parameters. As expected, we observe that there exists a limit to how much the crystal can be tilted if we want to satisfy the condition $\tilde{\Delta}<$ 0.1 ( $\sim 6$ degrees in the example), which imposes restrictions on techniques such as angular phase matching [34]; similarly, this condition imposes restrictions on the astigmatism of the mirror (an ellipticity below $\sim 0.1 \%$, in the example), although this is not a strong limitation since nowadays commercial mirrors can be made virtually perfectly spherical.

In the limit of small $\beta$ and $\varepsilon$, that is, small anisotropy, one can find a simple expression for the detuning to the leading order in these parameters:

$$
\tilde{\Delta}=\frac{2}{\mathcal{T}}\left[\frac{2 l_{\mathrm{c}}\left(n_{\mathrm{c}}^{2}-1\right)}{R n_{\mathrm{c}}^{3} \sqrt{1-g^{2}}} \beta^{2}+\sqrt{\frac{1-g}{1+g}}|\varepsilon|\right],
$$




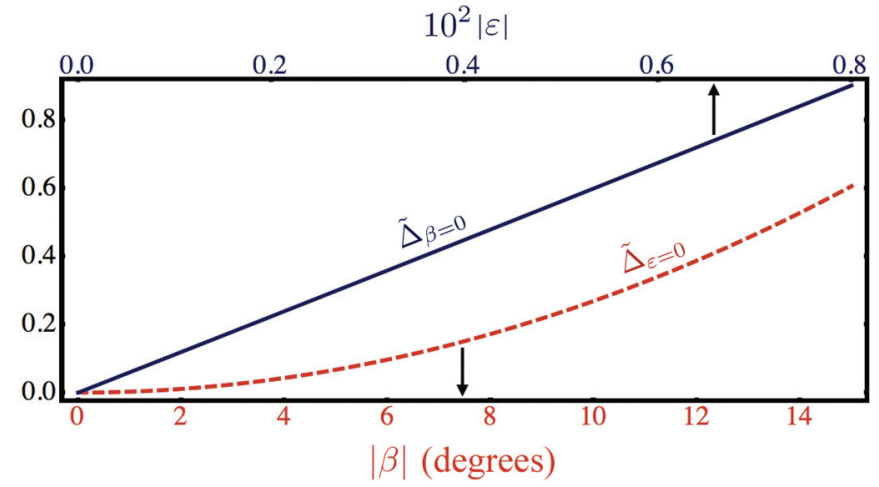

FIG. 2. (Color online) Normalized detuning $\tilde{\Delta}$ as a function of the tilting angle of the nonlinear crystal $\beta$ and the ellipticity of the astigmatic mirror $\varepsilon$. The solid and dashed lines correspond, respectively, to the case of a perfectly aligned crystal and a perfectly spherical mirror. Parameters are as follows: $R=R_{x}=2 L, l_{\mathrm{c}}=0.1 L$, $n_{\mathrm{c}}=2$, and $\mathcal{T}=0.01$.

where we have taken $R_{x}=R$ to simplify the expression, $g$ is the $g$ parameter of the cavity in the absence of anisotropy, and we have used $\gamma_{\mathrm{s}}=c \mathcal{T} / 4 L_{\text {opt }}$, being $\mathcal{T}$ the transmissivity of the output-coupling mirror.

Connection to the orientation's uncertainty. So far we have described the down-converted field in terms of two TEM modes aligned along the Cartesian axes $x$ (bright mode) and $y$ (dark mode). However, as the dark mode is not empty, one can partially visualize its effect as giving rise to a bright mode slightly rotated with respect to the $x$ axis, say by an angle $\theta(t)$. This leads to an alternative way to analyze the OPO dynamics in which this angle is treated as a dynamical variable $[18,20]$. This picture will provide an enlightening relationship between the bright mode's orientation indeterminacy and the dark mode's squeezing level. We include $\theta(t)$ into the description by writing the stochastic amplitudes as $[18,20]$

$$
\begin{aligned}
& \alpha_{x}(t)=\rho \cos \theta(t)+b_{x}(t) \cos \theta(t)+b_{y}(t) \sin \theta(t), \\
& \alpha_{y}(t)=\rho \sin \theta(t)+b_{x}(t) \sin \theta(t)+b_{y}(t) \cos \theta(t),
\end{aligned}
$$

and similarly for the $\alpha_{m}^{+}$amplitudes. The difference with respect to our previous works is that, in addition to the fluctuations $b_{m}$ and the orientation's time derivative $\dot{\theta}$, now we can take the orientation $\theta$ itself as a small quantity, as it is classically locked to $\theta=0$; we will come back to this point at the end of this section. Linearization of (1a) with respect to these variables leads to $\alpha_{x} \approx \rho+b_{x}$ and $\alpha_{y}=\rho \theta+b_{y}$, and hence all the information about the orientation $\theta$ is contained in the dark mode's evolution equations $(5 \mathrm{c})$, which, defining $\mathbf{b}_{y}=\operatorname{col}\left(b_{y}, b_{y}^{+}\right)$, can be written as

$$
\rho \mathbf{u}_{0} \dot{\theta}+\dot{\mathbf{b}}_{y}=-i \rho \Delta \mathbf{u}_{1} \theta-\mathcal{L} \mathbf{b}_{y}+\sqrt{\gamma_{s}} \eta_{y}(t),
$$

with $\mathbf{u}_{0}=\operatorname{col}(1,1)$ and $\mathbf{u}_{1}=\operatorname{col}(1,-1)$. Now, projecting this linear system onto $\mathbf{u}_{0}$ and $\mathbf{u}_{1}$, and calling $c_{j}=\mathbf{u}_{j} \cdot \mathbf{b}_{y}$ (we take $c_{0}=0$ as usual $[18,20]$ to compensate for the excess of variables), we get the linear system,

$$
\dot{\mathbf{x}}=-\mathcal{M} \mathbf{x}+\sqrt{2 \gamma_{\mathrm{s}}} \boldsymbol{\eta}(t),
$$

with

$$
\mathbf{x}=\left(\begin{array}{c}
2 \rho \theta \\
c_{1}
\end{array}\right), \quad \text { and } \quad \mathcal{M}=\left(\begin{array}{cc}
0 & i \Delta \\
i \Delta & 2 \gamma_{\mathrm{s}}
\end{array}\right),
$$

and where $\eta(t)$ contains two real, independent noises which satisfy the usual statistical properties (6). It is then very simple from this equation, for example, by diagonalizing $\mathcal{M}$, to obtain the following long-term variance for the bright mode's orientation,

$$
V_{\theta}^{\infty}=\left\langle\theta^{2}(t \rightarrow \infty)\right\rangle=1 / 2 \rho^{2} \tilde{\Delta}^{2},
$$

which shows that the quantum indetermination of the bright mode's orientation monotonically decreases with the level of anisotropy, that is, the squeezing of the dark mode is degraded as the orientation gets less uncertain, just as we expected. Note that we have assumed that $\theta$ does not make large excursions from $\theta=0$, that is, this expression is valid as long as $V_{\theta}^{\infty} \ll 1$; note, however, that $\rho^{2}$ approximately gives the number of photons contained in the bright mode, and hence the small- $\theta$ approximation is consistent even for small normalized detunings $\tilde{\Delta}$ as long as one works sufficiently above threshold.

Conclusions. In this brief report we have analyzed the impact that transverse anisotropy has onto the quantum properties of the two-transverse-mode DOPO [18,20], showing that a small amount of it can serve to lock the orientation of the bright and dark modes (very beneficial for experiments) while still allowing for large levels of noncritical squeezing in the dark mode.

\section{ACKNOWLEDGMENTS}

We thank Eugenio Roldán for fruitful discussions. This work has been supported by the Spanish Government and the European Union FEDER through Project No. FIS2011-26960. C.N.-B. acknowledges financial support from the Future and Emerging Technologies (FET) programme within the Seventh Framework Programme for Research of the European Commission under the FET-Open grant agreement MALICIA, Grant No. FP7-ICT-265522, and the Alexander von Humboldt Foundation through its Fellowship for Postdoctoral Researchers.
[1] K. Goda, O. Miyakawa, E. E. Mikhailov, S. Saraf, R. Adhikari, K. McKenzie, R. Ward, S. Vass, A. J. Weinstein, and N. Mavalvala, Nat. Phys. 4, 472 (2008).

[2] H. Vahlbruch, S. Chelkowski, B. Hage, A. Franzen, K. Danzmann, and R. Schnabel, Phys. Rev. Lett. 95, 211102 (2005).
[3] N. Treps, U. Andersen, B. Buchler, P. K. Lam, A. Maître, H.-A. Bachor, and C. Fabre, Phys. Rev. Lett. 88, 203601 (2002).

[4] N. Treps, N. Grosse, W. P. Bowen, C. Fabre, H.-A. Bachor, and P. K. Lam, Science 301, 940 (2003). 
[5] S. L. Braunstein and P. van Loock, Rev. Mod. Phys. 77, 513 (2005).

[6] C. Weedbrook, S. Pirandola, R. García-Patrón, N. J. Cerf, T. C. Ralph, J. H. Shapiro, and S. Lloyd, Rev. Mod. Phys. 84, 621 (2012).

[7] M. Mehmet, S. Ast, T. Eberle, S. Steinlechner, H. Vahlbruch, and R. Schnabel, Opt. Express 19, 25763 (2011).

[8] T. Eberle, S. Steinlechner, J. Bauchrowitz, V. Händchen, H. Vahlbruch, M. Mehmet, H. Müller-Ebhardt, and R. Schnabel, Phys. Rev. Lett. 104, 251102 (2010).

[9] M. Mehmet, H. Vahlbruch, N. Lastzka, K. Danzmann, and R. Schnabel, Phys. Rev. A 81, 013814 (2010).

[10] H. Vahlbruch, M. Mehmet, S. Chelkowski, B. Hage, A. Franzen, N. Lastzka, S. Gossler, K. Danzmann, and R. Schnabel, Phys. Rev. Lett. 100, 033602 (2008).

[11] Y. Takeno, M. Yukawa, H. Yonezawa, and A. Furusawa, Opt. Express 15, 4321 (2007).

[12] P. Meystre and D. F. Walls (eds.), Nonclassical Effects in Quantum Optics (American Institute of Physics, New York, 1991).

[13] G. J. de Valcárcel, G. Patera, N. Treps, and C. Fabre, Phys. Rev. A 74, 061801 (2006).

[14] G. Patera, N. Treps, C. Fabre, and G. J. de Valcárcel, Eur. Phys. J. D 56, 123 (2010).

[15] G. Patera, C. Navarrete-Benlloch, G. J. de Valcárcel, and C. Fabre, Eur. Phys. J. D 66, 241 (2012).

[16] I. Pérez-Arjona, E. Roldán, and G. J. de Valcárcel, Europhys. Lett. 74, 247 (2006).

[17] I. Pérez-Arjona, E. Roldán, and G. J. de Valcárcel, Phys. Rev. A 75, 063802 (2007).

[18] C. Navarrete-Benlloch, E. Roldán, and G. J. de Valcárcel, Phys. Rev. Lett. 100, 203601 (2008).

[19] C. Navarrete-Benlloch, G. J. de Valcárcel, and E. Roldán, Phys. Rev. A 79, 043820 (2009).

[20] C. Navarrete-Benlloch, A. Romanelli, E. Roldán, and G. J. de Valcárcel, Phys. Rev. A 81, 043829 (2010).

[21] C. Navarrete-Benlloch, E. Roldán, and G. J. de Valcárcel, Phys. Rev. A 83, 043812 (2011).

[22] F. V. Garcia-Ferrer, C. Navarrete-Benlloch, G. J. de Valcárcel, and E. Roldán, Opt. Lett. 35, 2194 (2010).
[23] C. Navarrete-Benlloch, Ph.D. thesis, Universitat de València, 2011.

[24] M. Lassen, G. Leuchs, and U. L. Andersen, Phys. Rev. Lett. 102, 163602 (2009).

[25] J. Janousek, K. Wagner, J.-F. Morizur, N. Treps, P. K. Lam, C. C. Harb, and H.-A. Bachor, Nat. Photon. 3, 399 (2009).

[26] B. Chalopin, F. Scazza, C. Fabre, and N. Treps, Phys. Rev. A 81, 061804(R) (2010).

[27] S. Reynaud, C. Fabre, and E. Giacobino, J. Opt. Soc. Am. B 4, 1520 (1987).

[28] A. Heidmann, R. J. Horowicz, S. Reynaud, E. Giacobino, C. Fabre, and G. Camy, Phys. Rev. Lett. 59, 2555 (1987).

[29] J. Laurat, L. Longchambon, C. Fabre, and T. Coudreau, Opt. Lett. 30, 1177 (2005).

[30] A. S. Villar, L. S. Cruz, K. N. Cassemiro, M. Martinelli, and P. Nussenzveig, Phys. Rev. Lett. 95, 243603 (2005).

[31] A. S. Villar, K. N. Cassemiro, K. Dechoum, A. Z. Khoury, M. Martinelli, and P. Nussenzveig, J. Opt. Soc. Am. B 24, 249 (2007).

[32] A. S. Villar, M. Martinelli, C. Fabre, and P. Nussenzveig, Phys. Rev. Lett. 97, 140504 (2006).

[33] A. S. Coelho, F. A. S. Barbosa, K. N. Cassemiro, A. S. Villar, M. Martinelli, and P. Nussenzveig, Science 326, 823 (2009).

[34] R. W. Boyd, Nonlinear Optics (Academic Press, San Diego, 2003).

[35] J. A. Fleck, Jr. and M. D. Feit, J. Opt. Soc. Am. 73, 920 (1983).

[36] M. Martinelli, J. A. O. Huguenin, P. Nussenzveig, and A. Z. Khoury, Phys. Rev. A 70, 013812 (2004).

[37] B. Coutinho dos Santos, A. Z. Khoury, and J. A. O. Huguenin, Opt. Lett. 33, 2803 (2004).

[38] B. Coutinho dos Santos, K. Dechoum, and A. Z. Khoury, Phys. Rev. Lett. 103, 230503 (2009).

[39] D. C. Hanna, IEEE J. Quantum Electron. 5, 483 (1969).

[40] N. Hodgson and H. Weber, Laser Resonators and Beam Propagation (Springer-Verlag, New York, 2005); A. E. Siegman, Lasers (University Science Books, Mill Valley, 1986).

[41] C. M. Savage and D. F. Walls, J. Opt. Soc. Am. B 4, 1514 (1987).

[42] C. Fabre, E. Giacobino, A. Heidmann, L. Lugiato, S. Reynaud, M. Vadacchino, and W. Kaige, Quantum Opt. 2, 159 (1990).

[43] M. J. Collett and D. F. Walls, Phys. Rev. A 32, 2887 (1985). 